\title{
POISSON BOUNDARIES AND ENVELOPES OF DISCRETE GROUPS
}

\author{
BY HARRY FURSTENBERG ${ }^{1}$
}

\author{
Communicated by G. D. Mostow, January 5, 1967
}

In [4] we defined the Poisson boundaries for semisimple Lie groups. These spaces play a role in the theory of generalized harmonic functions on the Lie group similar to that played by the boundary of the unit disc in the classical theory of harmonic functions on the unit disc. It is not hard to extend these notions to all separable, locally compact groups, and, in particular, they make sense for countable discrete groups. In this form we shall show that these ideas provide a useful tool for answering certain purely algebraic questions. Namely, we raise the following question. Let $G$ be a connected Lie group, $\Gamma$ a discrete subgroup for which $G / \Gamma$ has finite (left-) invariant measure. To what extent is $G$ determined by a knowledge of $\Gamma$ as an abstract group, and conversely, what is the influence of $G$ on the structure of $\Gamma$ ?

To make this question precise, let us say that $G$ is an envelope of $\Gamma$ if an isomorphic copy $\Gamma^{\prime}$ of $\Gamma$ occurs as a discrete subgroup of $G$, and $G=D \Gamma^{\prime}$, where $D$ is a subset of $G$ with finite left-invariant Haar measure. Our question may now be stated in this way. How different can two connected Lie groups $G_{1}$ and $G_{2}$ be if they both envelop the same countable group $\Gamma$ ?

We shall be discussing a rather restricted version of this question. We suppose that $G_{1}$ and $G_{2}$ are semisimple and have no compact components, and that $G_{1}$ and $G_{2}$ envelop the same group $\Gamma$. Does it follow that $G_{1}$ and $G_{2}$ are isomorphic? (Without the hypothesis that $G_{1}$ and $G_{2}$ have no compact components we could always take $G_{2}=G_{1} \times$ a compact group.) Our guess is that this is the case. However all we can prove is the following:

TheOREM. Let $H_{r}, r=1,2,3, \cdots$, denote the hyperbolic group of motions of the $r$-sphere $S^{r}: H_{r}$ consists of the $(r+2) \times(r+2)$ real matrices that leave the form $x_{0}^{2}+x_{1}^{2}+\cdots+x_{r}^{2}-t^{2}$ invariant. $S L(s, R)$ denotes the group of $s \times s$ real unimodular matrices. If $G_{1}$ is one of the groups $H_{r}, r \geqq 1$ and $G_{2}$ is one of the groups $S L(s, R), s \geqq 3$, then $G_{1}$ and $G_{2}$ cannot simultaneously envelop the same countable group.

1 This research was partially supported by the Office of Scientific Research of the Office of Aerospace Research of the USAF under Grant \#AF-AFOSR-381-63. 
Note that $S L(2, R)$ is exceptional here. This is because $S L(2, R)$ is isomorphic to the hyperbolic group $H_{1}$. (Consider the adjoint representation of $S L(2, R)$.) Thus, as a special case of the theorems, $S L(2, R)$ and $S L(s, R)$ cannot envelop the same countable group, if $s \geqq 3$.

Our theorem is rather special, but it implies, for example, that the free group on two generators which occurs with finite index in $S L(2, Z)$, cannot be enveloped by any $S L(s, R), s \geqq 3$. Actually we can say more: $S L(s, R), s \geqq 3$, cannot envelop any free group. In particular, no subgroup of $S L(s, \boldsymbol{Z}), s \geqq 3$, of finite index is free. This last result can also be deduced from [1] and [6].

We present here the main ideas of the proof of this theorem. A more detailed discussion of a partial result is given in [5].

1. The Poisson boundary. Let $G$ be a separable, locally compact group, $M$ a $G$-space, $\mu$ a probability (Borel) measure on $G$, and $\nu$ a probability measure on $M$. The application $G \times M \rightarrow M$ defines the convolution $\mu * \nu$, and we say that $\nu$ is a $\mu$-stationary measure if $\mu * \nu=\nu$. A bounded Borel function $f(g)$ on $G$ is $\mu$-harmonic if

$$
f(g)=\int f\left(g g^{\prime}\right) d \mu\left(g^{\prime}\right) .
$$

If $\nu$ is a $\mu$-stationary measure on $M, \phi$ a bounded Borel function on $M$, then

$$
f(g)=\int \phi(g \xi) d \nu(\xi)=\int \phi(\xi) d g \nu(\xi)
$$

defines a $\mu$-harmonic function on $G$.

Theorem 1. Let $\mu$ be a fixed probability measure on $G$. There exists $a G$-space $B$ and a $\mu$-stationary measure $\nu$ on $B$ such that every bounded $\mu$-harmonic function on $G$ admits the Poisson representation (1) for some function $\phi$ on $B$. The pair $(B, \nu)$ is called the Poisson boundary of $(G, \mu)$ and we write $(B, \nu)=P(G, \mu)$.

Let $X_{1}, X_{2}, \cdots, X_{n}, \cdots$ denote a sequence of independent $G$ valued random variables, each with distribution $\mu$. If $M$ is a $G$-space and $\nu$ a $\mu$-stationary measure on $M$, it may be shown that with probability 1 , the sequence of measures $\left\{X_{1} X_{2} \cdots X_{n} \nu\right\}$ converges weakly on $M$, as $n \rightarrow \infty$.

Definition 1 . The pair $(M, \nu)$ is a boundary of $(G, \mu)$ if, with probability $1, \lim X_{1} X_{2} \cdots X_{n} \nu$ is a point measure (i.e. its support consists of a single point). 
TheOREM 2. Every boundary of $(G, \mu)$ is a measurable equivariant image of the Poisson boundary $P(G, \mu)$.

That is, if $P(G, \mu)=\left(B, \nu_{0}\right)$, then there is an equivariant, measurable map $\rho: B \rightarrow M$ such that $\rho\left(\nu_{0}\right)=\nu$.

In case $G$ is a semisimple Lie group and $\mu$ is an absolutely continuous measure on $G$, we found in [4] that the underlying space $B$ of $P(G, \mu)$ is a compact homogeneous space of $G$. In fact, it must be one of finitely many covering spaces of the homogeneous space $B(G)$ which can be explicitly described: If $G=K \cdot A \cdot N$ is an Iwasawa decomposition of $G$ relative to the maximal compact subgroup $K$, then $B(G)=G / T$, where $T$ is the normalizer in $G$ of $A \cdot N$. Note that $K T=G$, so that $K$ is transitive on $B(G)$.

In certain cases we can be even more specific. We say $\mu$ is spherical if it is invariant with respect to left and right multiplication by elements of $K$. If $\mu$ is spherical, then $P(G, \mu)=\left(B(G), m_{B}\right)$, where $m_{B}$ denotes the unique $K$-invariant probability measure on $B(G)$. By Theorem 1 , it follows that all spherical measures $\mu$ lead to the same class of $\mu$-harmonic functions. We call these simply harmonic functions on $G$. (They do depend, however, on the choice of $K$.)

\section{Measures on discrete subgroups.}

TheOREM 3. If $G$ is a connected semisimple Lie group, $\Gamma$ a countable discrete subgroup enveloped by $G$, then there exists a measure $\mu$ whose support coincides with $\Gamma$ for which $P(\Gamma, \mu)=\left(B(G), m_{B}\right)$.

According to Theorem 3 , the Poisson representation of a $\mu$-harmonic function on $\Gamma$ coincides with that of a harmonic function on $G$. In fact, the theorem implies that the $\mu$-harmonic functions on $\Gamma$ are precisely the restrictions of harmonic functions from $G$ to $\Gamma$.

Theorem 3 provides the main tool for our subsequent analysis. It shows in what form one can obtain information about an envelope of $\Gamma$ from $\Gamma$ itself. For, the possible Poisson boundaries $P(\Gamma, \mu), \mu$ a measure on $\Gamma$, depend only on the structure of $\Gamma$.

3. Dynkin spaces and D-groups. Let $H$ be a locally compact topological group and $M$ an $H$-space which is compact and metrizable.

Definition 2. $M$ is a Dynkin space of $H$ if, for every $\epsilon>0$, there is a compact subset of $H$ such that each $h \in H$ outside of this set maps all of $M$ except for some $\epsilon$-neighborhood into an $\epsilon$-neighborhood:

For example, the projective line $P^{1}$ is a Dynkin space of $S L(2, R)$. More generally,

Proposition 1. The r-dimensional sphere is a Dynkin space of the hyperbolic group $H_{r}, r=1,2,3, \cdots$. 
Definition 3. A group $H$ is a D-group if it possesses a Dynkin space $M$ with the property that no point of $M$ is a fixed-point of the group.

$H_{r}$ is a $D$-group. The same is true of any subgroup of $H_{r}$ that has no fixed-point on the $r$-sphere. Since the subgroup that leaves a given point of $S^{r}$ fixed is connected, it follows from [2] that a closed subgroup $H \subset H_{r}$ for which $H_{r} / H$ has finite measure cannot have a fixedpoint on $S^{r}$. Hence

Proposition 2. If $\Gamma$ is enveloped by a hyperbolic group, then $\Gamma$ is a D-group.

For example, $S L(2, \boldsymbol{Z})$ and its subgroups of finite index are Dgroups. The fundamental groups of compact orientable 2-dimensional surfaces of genus $\geqq 2$ are D-groups. The Picard group of $2 \times 2$ unimodular matrices whose entries are Gaussian integers is enveloped by $S L(2, C)$ which is isomorphic to the Lorentz group $H_{2}$. Hence the Picard group is a D-group. We can obtain many other examples using the following proposition.

Proposition 3. $H$ is a D-group if some homomorphic image of $H$ is a D-group.

For example, the commutator subgroup of $S L(2, \boldsymbol{Z})$ is of finite index and is a free group with two generators. Now any free group on $\geqq 2$ generators maps homomorphically on to the latter. This gives us

Proposition 4. The free group on $r$ generators, $2 \leqq r \leqq \infty$, is a Dgroup.

Actually we may construct a Dynkin space for the free group $F_{2}$ on 2 generators directly as follows. Denote the generators by $a$ and $b$. Let $M$ be the set of all infinite "words," $w=w_{1} w_{2} \cdots w_{n} \ldots$ whose letters are chosen from $\left\{a, a^{-1}, b, b^{-1}\right\}$ and subject to the condition that no consecutive pair $\left(w_{i}, w_{i+1}\right)$ is of the form $\left(a, a^{-1}\right),\left(b, b^{-1}\right)$, $\left(a^{-1}, a\right)$, or $\left(b^{-1}, b\right) . M$ is a $F_{2}$-space if we define the action of $F_{2}$ by juxtaposition, cancelling where necessary. With the usual topology of a sequence space, $M$ is compact and metrizable. Moreover one sees quite easily that $M$ is a Dynkin space for $F_{2}$. This construction is essentially due to Dynkin and Malyutov [3] who use it to construct a Martin boundary for a class of harmonic functions on the free group.

A similar construction may be applied to other instances of groups presented in terms of generators and relations. The following is an example of this. 
Proposition 5. A free product of nontrivial finite groups, $G_{1}, G_{2}, \cdots, G_{m}(2 \leqq m \leqq \infty)$ is a D-group unless $m=2$ and $G_{1}$ and $G_{2}$ are both of order 2 .

Here the Dynkin space consists of words $w_{1} w_{2} \cdots w_{n} \cdots$ where the $w_{i}$ are nontrivial elements of the groups $G_{j}$ and where neighboring $w_{i}$ never come from the same group.

4. $\mu$-harmonic functions on a D-group. Let $\mu$ be a measure on a D-group $H$ whose support coincides with $H$, and suppose that $M$ is a Dynkin space for $H$. Since $M$ is compact, there exists a $\mu$-stationary measure $\nu$ on $M . \nu$ cannot concentrate on a single point of $M$, for then $H$ would have a fixed-point in $M$. Let us assume that, in fact, $\nu$ is entirely nonatomic. Then $(M, \nu)$ is a boundary of $(H, \mu)$. This follows from Definition 2, since, if $h_{n} \nu$ converges to a measure on $M$ and $h_{n} \rightarrow \infty$ in $H$, then $\lim h_{n} \nu$ must be a point measure. Now it can be shown that, with probability 1 , the sequence $\left\{X_{1} X_{2} \cdots X_{n}\right\}$ possesses a subsequence $\rightarrow \infty$ unless $\mu$ is concentrated on a compact subgroup of $H$. Thus, in our case, $\lim X_{1} X_{2} \cdots X_{n} \nu$ is a point measure, and $(M, \nu)$ is a boundary of $(H, \mu)$.

Now suppose $A_{1}$ and $A_{2}$ are two disjoint closed subsets of $M$ with $\nu\left(A_{i}\right)>\frac{1}{2}-\epsilon$, where $\epsilon$ is a positive number. The functions

$$
f_{i}(h)=\nu\left(h^{-1}\left(A_{i}\right)\right)=h \nu\left(A_{i}\right)
$$

are $\mu$-harmonic and $f_{i}(e)>\frac{1}{2}-\epsilon$. Now as $h \rightarrow \infty$, the measure $h \nu$ tends to become a point measure, and since $A_{1}$ and $A_{2}$ are separated by a positive distance, we find

$$
\min \left\{f_{1}(h), f_{2}(h)\right\} \rightarrow 0
$$

as $h \rightarrow \infty$. This gives us the following result.

LEMMA 1. If $H$ is a D-group and $\mu$ a probability measure on $H$ with support all of $H$, then either there exists an atomic $\mu$-stationary measure on the Dynkin space of $H$, or, for every $\epsilon>0$, there exist $\mu$-harmonic functions $f_{1}(h), f_{2}(h)$ on $H$ with

(a) $0 \leqq f_{1}(h), f_{2}(h) \leqq 1$

(b) $f_{1}(e)>\frac{1}{2}-\epsilon, f_{2}(e)>\frac{1}{2}-\epsilon$

(c) $\min \left\{f_{1}(h), f_{2}(h)\right\} \rightarrow 0$ as $h \rightarrow \infty$.

5. Boundary behavior of harmonic functions on $S L(s, R)$. Let $s \geqq 3$ be fixed, set $G=S L(s, R), B=B(G)$. $B$ can be explicitly determined; it is the "flag space" of subspaces of all dimensions of $R^{s}$. $K$ will denote the orthogonal subgroup of $G$, and $m_{B}$ denotes the $K$ invariant measure on $B$. Let $\left\{g_{n}\right\}$ be a sequence in $G$ such that $g_{n} m_{B}$ 
converges to a measure $\pi$ on $B$. We then have the following version of the Fatou theorem.

Proposition 6. If $f(g)$ is a bounded harmonic function on $G$ corresponding to a boundary function $\hat{f}$ on $B$, then, as functions on $K$, the sequence $f\left(k g_{n}\right)$ converges in measure to a function $\hat{f}_{\pi}(k)$ which satisfies

(a) $\hat{f}_{\pi}(k)=\int_{M} \hat{f}(k \xi) d \pi(\xi)$ a.e.,

(b) $\int_{K} \hat{f}_{\pi}(k) d k=f(e)$.

If $\pi$ is a point measure, $\pi=\delta_{\xi_{0}}$, then $\hat{f}_{\pi}$ simply "lifts" $\hat{f}$ from $B$ to $K: \hat{f}_{\pi}(k)=\hat{f}\left(k \xi_{0}\right)$. For $s \geqq 3$ however, there always are limit measures $\pi$ which are not point measures, and Proposition 6 describes the boundary behavior near these points as well. The values of $\hat{f}_{\pi}$ are averages of values of $\hat{f}(\xi)$; we may expect, therefore, that even if $\hat{f}$ takes on only the two values 0 and 1 , the function $\hat{f}_{x}$ will take on intermediate values as well. This is made precise in the following.

Lemma 2. There exist two limit measures $\pi^{\prime}$ and $\pi^{\prime \prime}$ on $B$ and a positive constant $\eta$ such that if $f(g)$ is a harmonic function on $G$ with $0 \leqq f(g) \leqq 1$, and also $1 / 4 \leqq f(e) \leqq 3 / 4$, then

$$
\int \min \left(\hat{f}_{\pi}(k), 1-\hat{f}_{\pi}(k)\right) d k>\eta
$$

for either $\pi=\pi^{\prime}$ or $\pi=\pi^{\prime \prime}$.

In other words, unless $f(e)$ is close to either 0 or 1 , it is not possible for both $\hat{f}_{\pi^{\prime}}$ and $\hat{f}_{\pi^{\prime \prime}}$ to approximate characteristic functions.

A comparison of Lemmas 1 and 2 enables us to prove our main result.

ThEOREM 4. A unimodular group $S L(s, R), s \geqq 3$, cannot envelop a D-group.

Suppose $G=S L(s, R)$ envelopes the D-group $\Gamma$. Let $\mu$ be a measure on $\Gamma$ for which $P(\Gamma, \mu)=\left(B, m_{B}\right)$, let $M$ be the Dynkin space of $\Gamma$ and let $\nu$ be a $\mu$-stationary measure on $M$. Then $(M, \nu)$ is a boundary, and by Theorem 2 , there is an equivariant map from $\left(B, m_{B}\right)$ to $(M, \nu)$. This may be seen to imply that $\nu$ is nonatomic. Applying Lemma 1 , we find that the second alternative takes place, and we may find two $\mu$-harmonic functions $f_{1}, f_{2}$ on $\Gamma$ satisfying (a), (b), and (c). Since $P(\Gamma, \mu)=\left(B, m_{B}\right), f_{1}$ and $f_{2}$ extend to harmonic functions on $G$. Now we can no longer assert that, in general, $\min \left(f_{1}(g), f_{2}(g)\right) \rightarrow 0$ as $g \rightarrow \infty$ in $G$, but this will be the case if $g$ stays "sufficiently close" to $\Gamma$. Using a result of [7] regarding the ergodicity of flows in $G / \Gamma$, we may show 
that each of the measures $\pi^{\prime}, \pi^{\prime \prime}$ is the limit of a sequence of the form $\left\{g_{n} m_{B}\right\}$ such that

$$
\min \left(f_{1}\left(k g_{n}\right), f_{2}\left(k g_{n}\right)\right) \rightarrow 0
$$

in measure (of $K$ ) as $n \rightarrow \infty$. But then $\min \left(\hat{f}_{1, \pi}, \hat{f}_{2, \pi}\right)=0$ for $\pi=\pi^{\prime}$ and $\pi=\pi^{\prime \prime}$. Now

$$
\int \hat{f}_{1, \pi}(k) d k+\int \hat{f}_{2, \pi}(k) d k=f_{1}(e)+f_{2}(e)>1-2 \epsilon
$$

so that $\int\left|\left(1-\hat{f}_{1, \pi}\right)-\hat{f}_{2, \pi}\right| d k<2 \epsilon$. This gives

$$
\int \min \left(\hat{f}_{1, \pi}(k), 1-\hat{f}_{1, \pi}(k)\right) d k<2 \epsilon
$$

for both $\pi=\pi^{\prime}, \pi^{\prime \prime}$. This contradicts Lemma 2 if $2 \epsilon<\eta$ and $\epsilon<1 / 4$.

As a direct consequence of Theorem 4 we have

Theorem 5. If $\Gamma$ is a countable group enveloped by a unimodular group $S L(s, R), s \geqq 3$, then $\Gamma$ is not a free group, nor is it a free product of finite groups, nor is it enveloped by a hyperbolic group, nor does it have a homomorphic image with any of these properties.

We should remark that whereas free groups cannot occur as discrete subgroups of a group $S L(s, R), s \geqq 3$, in such a way that the quotient space has finite measure, if we remove either the finiteness condition or the discreteness condition, they certainly do occur. In fact, free groups occur as dense subgroups in any connected Lie group.

\section{REFERENCES}

1. H. Bass, M. Lazard and J.-P. Serre, Sous-groupes d'indice fini dans $S L(n, Z)$, Bull. Amer. Math. Soc. 70 (1964), 385.

2. A. Borel, Density properties for certain subgroups of semi-simple groups without compact components, Ann. of Math. 72 (1960), 179-188.

3. E. B. Dynkin and M. B. Malyutov, Random walk on groups with finitely many generators, Soviet Math. Dokl. 2 (1961), 399-402.

4. H. Furstenberg, A Poisson Formula for semi-simple Lie groups, Ann. of Math. 77 (1963), 335-386.

5. — "Random walks and discrete subgroups of Lie groups," to appear in Advances in probability and related topics.

6. J. L. Mennicke, Finite factor groups of the unimodular groups, Ann. of Math. 81 (1965), 31-37.

7. C. C. Moore, Ergodicity of flows on homogeneous spaces, Amer. J. Math. 88 (1966), 154-178.

The Hebrew University, Jerusalem 